\title{
Table of cases and quasi-judicial decisions
}

\section{CJEU DECISIONS}

A, M, and S Europe Ltd (155/79) ECLI:EU:C:1982:157, [1982] ECR 1575 84,85

Abdida (C-562/13) ECLI:EU:C:2014:2453 …............................................ 340

Acereda Herrera (C-466/04) ECLI:EU:C:2006:405, [2006] ECR I-5431 ...............46 AG2R Prévoyance (C-437/09) ECLI:EU:C:2011:112, [2011] ECR I-00973

Ahokainen (C-434/04) ECLI:EU:C:2006:609, [2006] ECR I-9171 _.......... 376, 378

Åkerberg Fransson (C-617/10) ECLI:EU:C:2013:105 100

Akzo Nobel Chemicals v Commission (C-550/07) ECLI:EU:C:2010:512, [2010] ECR I-08301.... 84,85

Alliance for Natural Health (C-154 and 155/04) ECLI:EU:C:2005:449, [2005] ECR I-6451

Altmark (C-280/00) ECLI:EU:C:2003:415, [2003] ECR I-7747... 30, 284, 286, 287

Ambulanz Glöckner (C-475/99) ECLI:EU:C:2001:577, [2001] ECR I-8089 269,277

AOK Bundesverband (C-264/01, C-306/01, C-354/01 and C-355/01) ECLI:EU:C:2004:150, [2004] ECR I-2493 269

Apothekerkammer des Saarlandes and Others (Doc Morris) (C-171/07 and C-172/07) ECLI:EU:C:2009:316, [2009] ECR I-4171 $33,302,479$

Aragonesa (C-1/90 and C-176/90) ECLI:EU:C:1991:327, [1991] ECR I-4151

Arnold André (C-434/02) ECLI:EU:C:2004:800, [2004] ECR I-11825 ..... 357, 425

AstraZeneca (C-457/10 P) ECLI:EU:C:2012:770 …...................... 274, 275, 276

Aventis Pasteur SA v OB (C-358/08) ECLI:EU:C:2009:744, [2010] 2 CMLR 16 $174,175,181,193$

Bacardi France (C-429/02) ECLI:EU:C:2004:432, [2004] ECR I-6613 _............ 375

Berger (C-636/11) ECLI:EU:C:2013:227. 402

Blanco Pérez and Chao Gómez (C-570/07 and 571/07) ECLI:EU:C:2010: 300, [2010] ECR I-04629 33, 102-3, 105, 106, 302, 332,

Booker (C-20/00 and C-64/00) ECLI:EU:C:2003:397, [2003] ECR 479,495 I-7411. 341,342

Bostock (C-2/92) ECLI:EU:C:1993:116, [1994] ECR I-955 .. 101

Boston Scientific Medizintechnik GmbH v AOK Sachsen-Anhalt - Die Gesundheitskasse (C-503/13 and 504/13) ECLI:EU:C:2015:148, [2015] 3 CMLR 173...............................173, 174, 176-9, 182, 188, 191, 194, 195, 196

British American Tobacco (C-491/01) ECLI:EU:C:2002:741, [2002] ECR I-11453 $354,356,357,366-7,425$

Brüstle (C-34/10) ECLI:EU:C:2001:523, [2011] ECR I-09821 „............96, 97, 208, Centro Europa 7 (C-380/05) ECLI:EU:C:2007:59, [2008] ECR I-00349. 210, 220 
von Chamier-Glisczinski (C-208/07) ECLI:EU:C:2009:455, [2009] ECR I-6095.

Commission and France v Ladbroke Racing (C-359/95 P)

ECLI:EU:C:1997:531, [1997] ECR I-6265

Commission v Austria (C-424/99) ECLI:EU:C:2001:642, [2001] ECR I-9285 ... 306

Commission v Austria (C-28/09) ECLI:EU:C:2011:854, [2011] ECR I-13525 $85,103,106$

Commission v Finland (C-229/00) ECLI:EU:C:2003:334, [2003] ECR I-5727 306

Commission v France (216/84) ECLI:EU:C:1988:81, [1988] ECR 793

Commission v France (EDF) (C-159/94) ECLI:EU:C:1997:501, [1997] ECR I-5851

Commission v France (C-1/00) ECLI:EU:C:2001:687, [2001] ECR I-9989

Commission v France (C-52/00) ECLI:EU:C:2002:252, [2002] ECR I-3827.

Commission v France (loi Evin) (C-262/02) ECLI:EU:C:2004:431, [2004] ECR I-6569.

Commission v France (C-512/08) ECLI:EU:C:2010:579, [2010] ECR I-8833

$46,52,75,76$

Commission v Germany (70/72) ECLI:EU:C:1973:87, [1973] ECR 813 ...........284

Commission v Germany (274/87) ECLI:EU:C:1989:51, [1989] ECR 229 ......... 424

Commission v Germany (C-141/07) ECLI:EU:C:2008:492, [2008] ECR I-6935.

Commission v Greece (C-154/00) ECLI:EU:C:2002:252, [2002] ECR I-3879.

Commission v Italy (C-531/06) ECLI:EU:C:2009:315, [2009] ECR I-4103 ..........33

Commission v Luxembourg (C-490/09) ECLI:EU:C:2011:34, [2011] ECR I-249

Commission v Poland (C-29/14) ECLI:EU:C:2015:379 ............................... 235

Commission v Portugal (C-255/09) ECLI:EU:C:2011:695, [2011] ECR I-10547.

Commission v Spain (C-404/00) ECLI:EU:C:2003:373, [2003] ECR I-6695......284

Commission v Spain (C-211/08) ECLI:EU:C:2010:340, [2010] ECR I-5267........46

Commission v United Kingdom (C-300/95) ECLI:EU:C:1997:255, [1997] ECR I-2649. $173,174,178,181,191,192$

Consten and Grundig v Commission (56/64 and 58/64) ECLI:EU:C:1966: 41, [1966] ECR 299

Coote v Granada Hospitality (C-185/97) ECLI:EU:C:1998:424, [1998] ECR I-05199

Corbeau (C-320/91) ECLI:EU:C:1993:198, [1993] ECR I-2533 _.....................277

Costa v ENEL (6/64) ECLI:EU:C:1964:66, [1964] ECR 585.............................8 84

Criminal Proceedings against Christina Bellamy and English Shop Wholesale (C-123/00) ECLI:EU:C:2001:214, [2001] ECR I-2795 ................424

Criminal Proceedings against Van der Veldt (C-17/93) ECLI:EU:C:1994: 299, [1994] ECR I-3537.

Decker v Caisse de maladie des employés privés (C-120/95)

ECLI:EU:C:1998:167, [1998] ECR I-1831

Defrenne v SABENA (43/75) ECLI:EU:C:1976:56, [1976] ECR 455 .................94

Defrenne v SABENA (149/77) ECLI:EU:C:1978:130, [1978] ECR 1365............85

Denkavit (C-507/99) ECLI:EU:C:2002:4, [2002] ECR I-169 333 
Deutsche Apothekerverband v DocMorris NV and Jacques Waterval (C322/01) ECLI:EU:C:2003:664, [2003] ECR I-14887

Deutsche Telekom (C-280/08) ECLI:EU:C:2010:603, [2010] ECR I-9555 ........ 271

Deutsches Weintor (C-544/10) ECLI:EU:C:2012:526 … 78, 99, 103, 104, 106, 387

Dynamic Medien (C-244/06) ECLI:EU:C:2008:85, [2008] ECR I-00505 ............. 84

Elchinov (C-173/09) ECLI:EU:C:2010:581, [2010] ECR I-8889 ........................46

Elliniki Radiophonia Tileorassi AE v Dimotiki Etairia Pliroforissis and Aotirios Kouvelas (C-260/89) ECLI:EU:C:1991:254, [1991] ECR I-02925 _....85

Estonia v European Parliament and Council (C-508/13) ECLI:EU:C:2015: 403

European Parliament v Council (C-540/03) ECLI:EU:C:2006:429, [2005] ECR I-05769

Expedia (C-226/11) ECLI:EU:C:2012:795

Federación Española de Empresas de Tecnología Sanitaria (FENIN) v Commission (C-205/03 P) ECLI:EU:C:2006:453, [2006] ECR I-6295

France v Commission (C-601/11 P) ECLI:EU:C:2013:465

Franzen (C-189/95) ECLI:EU:C:1997:504, [1997] ECR I-5909 375

Germany v European Parliament and Council (Tobacco Advertising I) (C-376/98) ECLI:EU:C:2000:544, [2000] ECR I-8419 ......27, 76, 199, 354, 355, $357,425,481,482$

Germany v European Parliament and Council (Tobacco Advertising II) (C-380/03) ECLI:EU:C:2006:772, [2006] ECR I-11573 $76,354,355,356$,

GlaxoSmithKline and others v Commission, Commission v GlaxoSmithKline and others (C-501/06 P, C-513/06 P, C-515/06 P and C-519/06 P) ECLI:EU:C:2009:610, [2009] ECR I-9291 $272,273,278$

González Sanchez v Medicina Asturiana SA (C-183/00) ECLI:EU:C:2002: 255, [2002] ECR I-3901

Gourmet International Products (C-405/98) ECLI:EU:C:2001:135, [2001] ECR I-1795 374,376

Grogan (C-159/90) ECLI:EU:C:1991:378, [1991] ECR I-4685.... 28,69

Hartlauer (C-169/07) ECLI:EU:C:2009:141, [2009] ECR I-1721

Heinonen (C-394/97) ECLI:EU:C:1999:308, [1999] ECR I-3599 75,302

Hoffmann-La Roche v Commission (85/76) ECLI:EU:C:1979:36, [1979] ECR 461 376

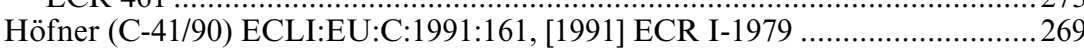
273

Human Plasma (C-421/09) ECLI:EU:C:2010:760, [2010] ECR I-12869 ............2239

IKA v Ioannidis (C-326/00) ECLI:EU:C:2003:101, [2003] ECR I-1703 .............46 Inizan (C-56/01) ECLI:EU:C:2003:578, [2003] ECR I-12403 ….....................46 International Stem Cell (C-364/13) ECLI:EU:C:2014:2451 ........ 97, 209, 210, 220,

Internationale Handelsgesellschaft (11/70) ECLI:EU:C:1970:114, [1970] ECR 1125 84

Inuit (C-583/11 P) ECLI:EU:C:2013:625 ................................................... 95

Ireland v Parliament and Council (C-301/06) ECLI:EU:C:2009:68, [2009] ECR I-593 354

Jaeger (C-151/02) ECLI:EU:C:2003:437, [2003] ECR I-8389 28

Jippes (C-189/01) ECLI:EU:C:2001:420, [2001] ECR I-5689 333,335

Ker-Optika v ANTSZ (C-108/09) ECLI:EU:C:2010:725, [2010] ECR I-12213 
Kohll v Union des caisses de maladie (C-158/96) ECLI:EU:C:1998:171, [1998] ECR I-01931. $30,45,58,59,74,199$

Konstantinides (C-168/91) EU:C:1992:115, [1993] ECR I-1191 ...............100-101

Kücükdeveci (C-555/07) ECLI:EU:C:2010:21, [2010] ECR I-365 ................. 333

Léger (C-528/13) ECLI:EU:C:2015:288 .................................103, 106, 333, 343

Leichtle (C-8/02) ECLI:EU:C:2004:161, [2004] ECR I-2641 ............................46

Les Laboratoires Servier (C-691/13) ECLI:EU:C:2015:121 …....................... 306

LFB and others (C-271/14 and C-273/14) ECLI:EU:C:2015:237 ….................. 306

Lindqvist (C-101/01) ECLI:EU:C:2003:596, [2003] ECR I-12971 _....................99

Luisi and Carbone v Ministero del Tesoro (286/82 and 26/83) ECLI:EU:C:

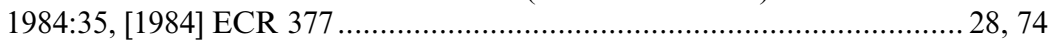

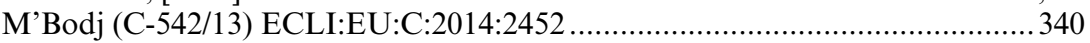

Mangold (C-144/04) ECLI:EU:C:2005:709, [2005] ECR I-09981 ....................... 94

Max Schrems v Data Protection Commissioner (C-362/14) ECLI:EU:C: 2015;650

Menarini (C-352/07 to C-356/07, C-365/07 to C-367/07 and C-400/07) ECLI:EU:C:2009:217, [2009] ECR I-2495

Müller-Faurè and van Riet (C-385/99) ECLI:EU:C:2003:270, [2003] ECR I-4509

Neptune Distribution Service (C-157/14) ECLI:EU:C:2015:823 ................... 388

Netherlands v Parliament and Council (C-377/98) ECLI:EU:C:2001:523, [2001] ECR I-7079 $88,89,95,493,494$

NFU (C-157/96) ECLI:EU:C:1998:191, [1998] ECR I-2211 …......................... 331

Nold KG v Commission (4/73) ECLI:EU:C:1974:51, [1974] ECR 491

Novo Nordisk AS v Ravimiamet (C-249/09) ECLI:EU:C:2011:272, [2011] ECR I-3155

NS and ME v Minister for Justice (C-411/10 and 493/10) ECLI:EU:C:2011: 865, [2011] ECR I-13905....

$\mathrm{O}$ and B v Minister voor Immigratie (C-456/12) ECLI:EU:C:2013:135 ............ 101

O’Byrne v Sanofi Pasteur MSD Limited (formerly Aventis Pasteur MSD Limited) (C-127/04) ECLI:EU:C:2006:93, [2006] 2 CMLR 24

Omega (C-36/02) ECLI:EU:C:2004:614, [2004] ECR I-09609.... 184,193

Österreicher Rundfunk (C-465/00, 138/01 and 139/01) ECLI:EU:C:2003: 294, [2003] ECR I-04989.

P v S \& Cornwall County Council (C-13/94) ECLI:EU:C:1996:170, [1996] ECR I-02143 ......................................................................... 85, 88

Pavlov (C-180/98 to 184/98) ECLI:EU:C:2000:428, [2000] ECR I-6451 ............269

Philip Morris (Tobacco Products II) (C-547/14) ECLI:EU:C:2016:325 .......... 354, $360,366,367,388,394$

Pierik (I) (117/77) ECLI:EU:C:1978:72, [1978] ECR 825 …...................... 73, 74 Pierik (II) (182/78) ECLI:EU:C:1979:142, [1979] ECR 1977 .................... 73, 74 Pillbox 38 (C-477/14) ECLI:EU:C:2016:324 ................................. 331, 344

Plaumann (25/62) ECLI:EU:C:1963:17, [1963] ECR 199 .................................95

Poland v European Parliament and Council (C-358/14) ECLI:EU:C:2015: 323 $103,104,342,425$

Procureur de la Republique v Gerard Tissier (35/85) ECLI:EU:C:1986:143, [1986] ECR 1207

Reynolds Tobacco and Others v Commission (C-131/03 P)

ECLI:EU:C:2006:541, [2006] ECR I-7795 354 
Rewe-Zentral AG v Bundesmonopolverwaltung für Branntwein (Cassis de Dijon) (120/78) ECLI:EU:C:1979:42, [1979] ECR 649 ............ 26, 68, 374, 375,

Rosengren (C-170/04) ECLI:EU:C:2007:313, [2007] ECR I-4071 .......... 375, 376, 377,378

Ruiz Zambrano (C-34/09) ECLI:EU:C:2010:124, [2011] ECR I-1177 .............. 101

Sabine Mayr (C-506/06) ECLI:EU:C:2008:119, [2008] ECR I-01017 ............... 209

Sandoz (174/82) ECLI:EU:C:1983:213, [1983] ECR 2445 .............................. 102

Schaible (C-101/12) ECLI:EU:C:2013:661 ..................... 330, 332, 337, 341, 342

Scotch Whisky Association (C-333/14) ECLI:EU:C:2015:845 ..........68, 374, 375,

$376,378,379-80$

Široká (C-459/13) ECLI:EU:C:2014:2120 335

Skov v Bilka (C-402/03) ECLI:EU:C:2006:6, [2006] 2 CMLR 16...................... 174

Smits and Peerbooms (C-157/99) ECLI:EU:C:2001:404, [2001] ECR I-5473. $30,45,333,344$

Sodemare SA and others v Regione Lombardia (C-70/95) ECLI:EU:C: 1997:301, [1997] ECR I-3395.

Sot. Lelos kai Sia EE (C-468/06 to 478/06) ECLI:EU:C:2008:504 ........... 273, 278

Stamatelaki (C-444/05) ECLI:EU:C:2007:231, [2007] ECR I-3185 79,101

Stauder v City of Ulm (29/69) ECLI:EU:C:1969:57, [1969] ECR 419.

Susisalo, Tuomaala and Ritala (C-84/11) ECLI:EU:C:2012: 374

Swedish Match (C-210/03) ECLI:EU:C:2004:802, [2004] ECR I-11893 …....... 354,

Test-Achats (C-236/09) ECLI:EU:2011:100, [2011] ECR I-00773 .....................98

United Brands v Commission (27/76) ECLI:EU:C:1978:22, [1978] ECR 207

United Kingdom v Commission (C-180/96) ECLI:EU:C:1998:192, [1998] ECR I-2265.

The Upjohn Company and Another v Farzoo Inc and Another (C-112/89) ECLI:EU:C:1991:147, [1991] ECR I-1703 157,158

Vanbraekel (C-368/98) ECLI:EU:C:2001:400, [2001] ECR I-5363 ....................45

Van Gend en Loos (26/62) ECLI:EU:C:1963:1, [1963] ECR 1 ........................8 84

Veedfald v Arhus Amtskommune (C-203/99) ECLI:EU:C:2001:258, [2003] 1 CMLR 41 184,193 Venturini (C-159/12 to 161/12) ECLI:EU:C:2013:791 103,106

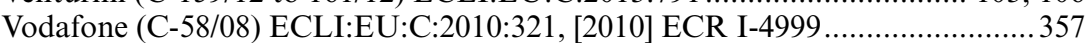

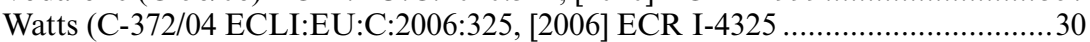

\section{CJEU OPINIONS}

Opinion 1/94 Opinion Pursuant To Article 228(6) of the EC Treaty (WTO Agreement) ECLI:EU:C:1994:384, [1994] ECR I-5267 433,456

Opinion 2/94 Accession by the Community to the European Convention for the Protection of Human Rights and Fundamental Freedoms EU:C:1996:140, [1996] ECR I-01759. 86

Opinion 2/13 Accession of the European Union to the European Convention for the Protection of Human Rights and Fundamental Freedoms ECLI:EU:C:2014:2454 


\section{GENERAL COURT DECISIONS}

Abad Pérez (T-304/01) ECLI:EU:T:2006:389, [2006] ECR II-4857 .......... 340, 341 Alpharma (T-70/99) ECLI:EU:T:2002:210, [2002] ECR II-3495 ............ 328, 329, $330,331,333,341$

ATC (T-333/10) ECLI:EU:T:2013:451 .......................................317, 340, 341 BUPA et al v Commission (T-289/03) ECLI:EU:T:2008:29, [2008] ECR II-81

CBI v Commission (T-137/10) ECLI:EU:T:2012:584. $287-8$

Coldiretti (T-149/96) ECLI:EU:T:1998:228, [1998] ECR II-3841 ............... 331, 340

É.R. (T-138/03) ECLI:EU:T:2006:39, [2006] ECR II-4923. 340

France v Commission (T-257/07 R) ECLI:EU:T:2007:300, [2011] ECR II5827

GlaxoSmithKline (T-168/01) ECLI:EU:T:2006:265, [2006] ECR II-2969

$272,273,278$

Kadi v Council and Commission (T-315/01) ECLI:EU:T:2005:332, [2005] ECR II-03649

Mannesmanröhren-werke v Commission (T-112/98) ECLI:EU:T:2001:61, [2001] ECR II-2233

\section{EFTA COURT OPINIONS}

Case E-01/94 Restamark (EFTA Court, 16 December 1994). 375

\section{COMMISSION COMPETITION DECISIONS}

APW/APSA/Nordic Capital/Capio (COMP/M.4367) Commission Decision of 16 March 2007.

Boots/Alliance Unichem (COMP/M.3990) Commission Decision of 30

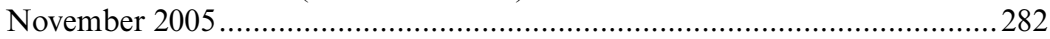

Bridgepoint Capital/Medi-Partenaires (COMP/M.7221) Commission Decision of 28 April 2014

Brocacef/Mediq Netherlands (COMP/M.7494) Commission Decision of 17 April 2015

CVC Capital Partners/Vedici Groupe (COMP/M.7321) Commission Decision of 5 August 2014

Fentanyl (COMP/AT.39685) Commission Decision of 10 December 2013

Fresenius/Helios (COMP/M.4010) Commission Decision of 8 December 2005

Ireland - Capital allowances for hospitals Commission (N543/2001)

Commission Decision of 27 February 2002

Johnson \& Johnson/Guidant (COMP/M.3687) Commission Decision of 25 August 2005

Lundbeck (COMP/AT.39226) Commission Decision of 19 June 2013 ...... 275, 276 
ONP (COMP/39.510) Commission Decision of 8 December 2010.......... 270, 271,

Perindopril (Servier) (COMP/AT.39612) Commission Decision of 9 July 2014

Pfizer/Hospira (COMP/M.3551) Commission Decision of 15 September 2004 282,283

Ramsay Healthcare/Credit Agricole/Generale De Sante (COMP/M.7322) Commission Decision of 4 August 2014 .......................................................2282

Retention of financial reserves by Dutch Health Insurance Funds (N541/2004) Commission Decision of 22 December 2005. 286

Vedici/Vitalia (COMP/M.7725) Commission Decision of 28 August 2015........2.282 Zorgverzekeringswet (N541/2004 and N542/2004) Commission Decision of 22 December 2005 286,287

\section{EPO DECISIONS}

Ciba Geigy, Technical board (T49/83) ECLI:EP:BA:1983:T004983.

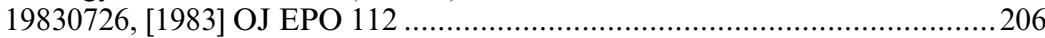

President and Fellows of Harvard College, Technical board (T19/90) ECLI: EP:BA:1993:T006592.19930613, [1990] OJ EPO 476 ….............................206

Relaxin, Examining Division (T67/94) ECLI:EP:BA:1994:T006794. 19941208, [1994] OJ EPO 388 206

\section{ECtHR CASES}

Bosphoros v Ireland App No 45036/98 (ECtHR, 30 June 2005). 86

Cantoni v France App No 17682/91 (ECtHR, 15 November 1996)...........................86 DSR-Senator Lines GmbH v the 15 Member States of the European Union App No 56672/00 (ECtHR, 10 March 2004) (admissibility decision) .............86

Enhorn v Sweden App No 56529/00 (ECtHR, 25 January 2005) ........................339

Glass v United Kingdom App No 61827/00 (ECtHR, 9 March 2004) ............... 169

Guérin Automobiles v Les 15 Etats de L'Union Européenne App No $51717 / 99$ (ECtHR, 4 July 2000) ................................................................ 86

Hristozov and Others v Bulgaria App No 47039/11 and 358/12 (ECtHR, 29

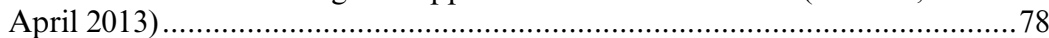

KH and others v Slovakia App No 32881/04 (ECtHR, 28 April 2009).............. 493

Matthews v UK App No 24833/94 (ECtHR, 18 February 1999).............................86

N v UK App No 26565/05 (ECtHR, 27 May 2008) .............................................340

Paposhvili v Belgium App No 41738/10 (ECtHR, 17 April 2014) ....................... 340

RR v Poland App No 27617/04 (ECtHR, 26 May 2011) ................................... 493

SEGI v the 15 Member States of the EU App Nos 6422/02 and 9916/02 (ECtHR, 23 May 2002) (admissibility decision).

SJ v Belgium App No 70055/10 (ECtHR, 19 March 2015)............................... 340

Tysiąc v Poland App No 5410/03 (ECtHR, 20 March 2007) 169,493 
xlii Research handbook on EU health law and policy

\section{INTERNATIONAL ARBITRATION DECISIONS}

Achmea B. V. v The Slovak Republic, UNCITRAL, PCA Case No 2008-13, Award (7 December 2012)

Achmea B. V. $v$ The Slovak Republic, UNCITRAL, PCA Case No 2013-12

(Number 2), Award (18 December 2014).

Eli Lilly and Company $v$ The Government of Canada, ICSID Case No

UNCT/14/2 (Ongoing).

Eureko B.V. v Republic of Poland, ad hoc Partial Award (19 August 2005)

Ioan Micula, Viorel Micula, S.C. European Food S. A, S.C. Starmill S. R.L. and S.C. Multipack S.R.L. v Romania, ICSID Case No ARB/05/20, Award (11 December 2013)

Philip Morris Asia Ltd v The Commonwealth of Australia, UNCITRAL, PCA Case No 2012-12, Award (17 December 2015).

Philip Morris Brands Sàrl, Philip Morris Products S. A. and Abal Hermanos S.A. v Oriental Republic of Uruguay, ICSID Case No ARB/10/7 (Ongoing)....

\section{WTO/GATT MATERIALS}

WTO, EC Measures Concerning Meat and Meat Products (Hormones), Complaint by the United States - Panel Report (13 February 1998) WT/ DS26/R/USA, as modified by Appellate Body Report (13 February 1998) WT/DS26/AB/R and WT/DS48/AB/R [1998] 3 DSR 699

WTO, EC Measures Concerning Meat and Meat Products (Hormones), Complaint by the United States - Appellate Body Report (13 February 1998) WT/DS26/AB/R and WT/DS48/AB/R [1998] 3 DSR 699

WTO, EC Measures Affecting Asbestos and Asbestos-Containing Products - Report of the Appellate Body (5 April 2001) WT/DS135/AB/R [2001] 7 DSR 3243

WTO, EC Certain Measures Affecting Poultry Meat and Poultry Meat Products from the United States - Request for Consultations (20 January 2009) WT/DS389/1

WTO, European Union and a Member State - Seizure of Generic Drugs in Transit - Request for Consultations (19 May 2010) WT/DS408/1

WTO, United States - Measures Affecting the Cross-Border Supply of Gambling and Betting Services.....

\section{NATIONAL CASES}

\section{X.i Austria}

OGH, 10ObS2303/96s, 12.09.1996 .64

VfGH G24/98 ua; V38/98 ua - V62/99, 18.03.2000 (Austrian Constitutional Court)

\section{X.ii Germany}

Bundesverfassungsgericht, 6.12.2005, 1 BvR 347/98 ('Nikolaus-

Beschluss') 
Bundesgerichtshof, 9.05.1995, VI ZR 158/94, 2 NJW 1995, 2162 ('Sparkling Water Bottle').

\section{X.iii Ireland}

Attorney General Ex rel Society for the Protection of Unborn Children (Ireland) Limited v Open Door Counselling Limited and the Welfare Dublin Wellwoman Centre Limited [1988] 2 CMLR 443

Society for the Protection of Unborn Children (Ireland) Limited v Stephen Grogan and Others [1990] 1 CMLR 689

\section{X.iv South Africa}

Minister of Health v Treatment Action Campaign (TAC) 2002 (10) BCLR 1075 (CC) (South Africa)....

\section{X.v United Kingdom}

A and others v National Blood Authority and another [2001] 3 All ER 289 (QB) $176,182,186,189,191,192$

BetterCare Group Ltd v Director General of Fair Trading [2002] CAT 7, [2002] Comp AR 299

Distillers Co (Biochemicals) Ltd v Thompson (Laura Anne) [1971] AC 458 (PC)

Montgomery v Lanarkshire Health Board (GMC intervening) [2015] AC 1430 (SC)

O’Byrne v Aventis Pasteur SA [2010] UKSC 23.

Pollard v Tesco Stores Limited [2006] EWCA Civ 393 (CA) ............. 186, 187, 189

R v Central Birmingham Health Authority, ex parte Collier (CA, 6 January 1988)

R v Department of Health, ex parte Source Informatics [2001] QB 424 (QB).....

R v Human Fertilisation and Embryology Authority, ex parte Blood [1997] 2 All ER 687 (CA)

The Scotch Whisky Association v Lord Advocate, Court of Session (Inner House) [2014] CSIH 38

\section{X.vi United States}

Brown v Superior Court 751 P.2d 470 (1988) (Supreme Court of California)

Diamond v Chakrabarty 206 USPQ 193 (1980)

Feldman v Lederle Laboratories (1984) 479 A.2d 374 (New Jersey Supreme Court)

Wyeth v Diana Levine 555 US 555 (2009) (US Supreme Court) 183 
Tamara K. Hervey, Calum A. Young, and Louise E. Bishop - 9781785364723 Downloaded from PubFactory at 04/25/2023 11:16:29PM 